\title{
M31 Planetary nebulae as seen by the Panchromatic Hubble Andromeda Treasury
}

\author{
Philip A. Rosenfield ${ }^{1}$, L. Clifton Johnson ${ }^{1}$, Léo Girardi ${ }^{2}$, Julianne J.
} Dalcanton $^{1}$, Benjamin F. Williams ${ }^{1}$, Luciana Bianchi ${ }^{3}$, Paola Marigo ${ }^{4}$, Nelson Caldwell ${ }^{5}$, Anil C. Seth ${ }^{6}$, Karl Gordon ${ }^{7}$, and Dustin Lang ${ }^{8}$ for the PHAT Team

\author{
${ }^{1}$ Department of Astronomy, University of Washington, \\ Box 351580, Seattle, WA 98195, USA \\ ${ }^{2}$ Osservatorio Astronomico di Padova - INAF, \\ Vicolo dell'Osservatorio 5, I-35122 Padova, Italy \\ ${ }^{3}$ Department of Physics and Astronomy, Johns Hopkins University, \\ Baltimore, MD 21218, USA \\ ${ }^{4}$ Dipartimento di Astronomia, Universitá di Padova, \\ Vicolo dell'Osservatorio 2, 35122 Padova, Italy \\ ${ }^{5}$ Harvard-Smithsonian Center for Astrophysics, \\ 60 Garden Street Cambridge, MA 02138, USA \\ ${ }^{6}$ Department of Physics \& Astronomy, University of Utah, \\ Salt Lake City, UT 84112, USA \\ ${ }^{7}$ Space Telescope Science Institute, \\ MD 21218, USA \\ ${ }^{8}$ Department of Astrophysical Sciences, Princeton University, \\ Princeton, NJ 08544, USA
}

\begin{abstract}
We present a preliminary analysis of known planetary nebulae (PNe) in M31 that were observed in the first year of the Panchromatic Hubble Andromeda Treasury HST Multicycle program. We use the properties of this sample to discuss PNe from this new multi-band survey.
\end{abstract}

Keywords. stars: evolution, stars: AGB and post-AGB, planetary nebulae: general, galaxies: individual (M31)

\section{Introduction}

The Andromeda Galaxy is the nearest giant galaxy and is rich with planetary nebulae (PNe; e.g., Ciardullo et al. 1989; Merrett et al. 2006). The Panchromatic Hubble Andromeda Treasury (PHAT) is a Hubble Space Telescope (HST) Multi-cycle program that will image roughly one third of M31's star-forming disk in six filters from UV to IR. As a result, PHAT will enable M31 to be a fundamental calibrator of stellar evolution and star-formation processes.

Although not primarily designed to study PNe, PHAT provides accurate photometry in 6 filters, including HST/ACS F475W, which covers the [O III] $5007 \AA$ emission line. Thus, the PHAT survey offers the wavelength coverage, the large spatial resolution, and the high astrometric accuracy needed to study M31's PNe population across the radial extent of its bulge and disk.

We briefly describe the results from a preliminary analysis of PNe observed in the first year of the PHAT survey. 


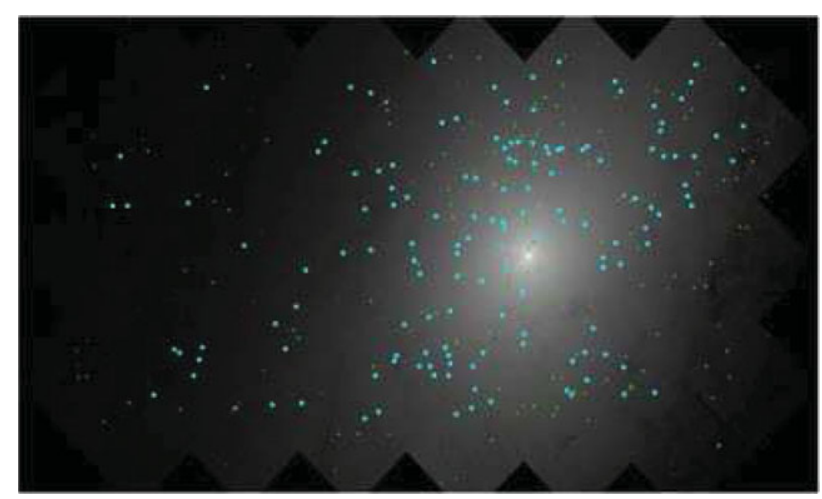

Figure 1. Spatial distribution of M06 Cataloged PNe (green) and sources matched to PHAT photometry (cyan) overlaid on a F475W image of a $2.6 \times 1.4 \mathrm{kpc}^{2}$ region of M31's bulge.

\section{PHAT data}

We analyzed some of the first data available from the PHAT program (GO-12058) consisting of UV, optical, and IR imaging two $3 \times 6$ arrays of HST pointings with a $180^{\circ}$ flip between each $3 \times 3$ subgrids. Each of these "bricks" corresponds to an area of $12^{\prime} \times 6.5^{\prime}\left(2.6 \times 1.4 \mathrm{kpc}^{2}\right.$; assuming a distance modulus of $(m-M)=24.47$ from McConnachie et al. 2005). Brick 1 includes the center of M31 and Brick 9 is a disk field with galactocentric radius $\sim 6.5 \mathrm{kpc}$. For full details of the PHAT observing strategy see Dalcanton et al. (in prep).

\section{Matching known PNe with PHAT sources}

Merrett et al. (2006, hereafter M06) provides the most extensive M31 PNe catalog to date, selected via low-dispersion spectra with the PNe Spectrograph. Their astrometry is good to $\sim 0.2$ arcsec. The catalog spatially covers the M31 disk up to $\sim 30 \mathrm{kpc}$, though it is incomplete in the inner $\sim 100 \mathrm{pc}$.

We cross-matched the PNe from the M06 catalog with the photometric catalogs for Bricks 1 and 9, (Fig. 1). The matched sources have mean positional offsets of $\Delta(\mathrm{RA})=$ $-0.59^{\prime \prime}$ and $\Delta(\mathrm{Dec})=-0.43^{\prime \prime}$ between M06 and PHAT catalogs (Fig. 2). One typical identification is illustrated in Fig. 3, where the PN is evident as the brightest object in $\mathrm{F} 475 \mathrm{~W}$; in these false-color $\mathrm{F} 336 \mathrm{~W}+\mathrm{F} 475 \mathrm{~W}+\mathrm{F} 814 \mathrm{~W}$ images, the PNe appear as green dots.
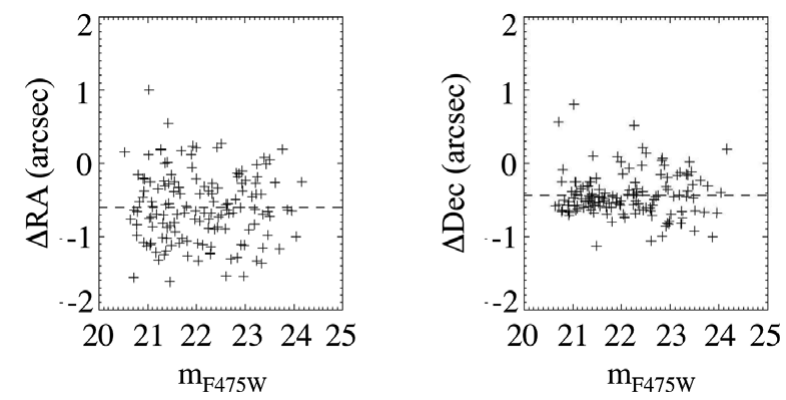

Figure 2. For 143 likely matches in Brick 1, RA/Dec offsets between PHAT and M06 positions $(\Delta=$ PHAT - M06). 


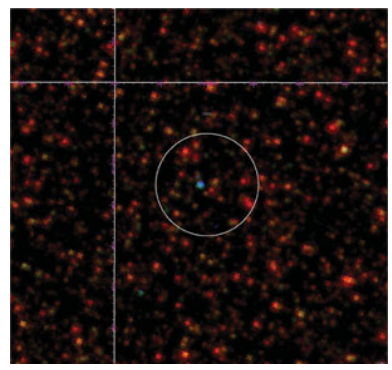

Figure 3. Example of a PNe observed in the outskirts of Brick 1. It appears as a greenish stellar-like object inside $1^{\prime \prime}$ radius circle around a M06 PN.
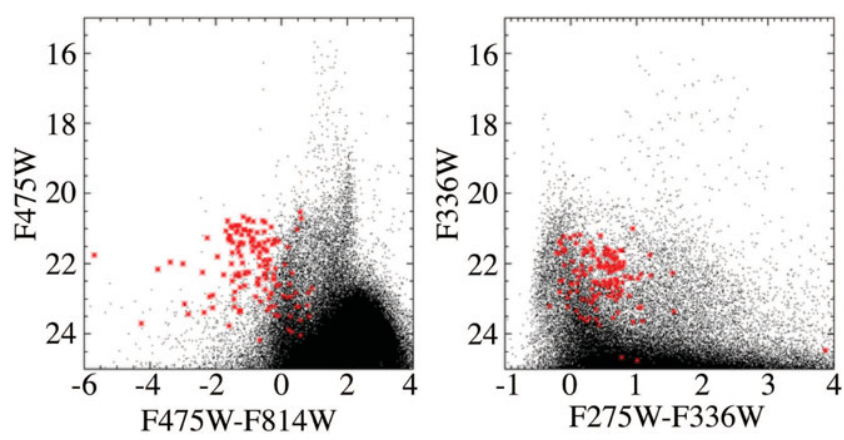

Figure 4. Distribution of Brick 1 PNe (asterisks) in two CMDs from PHAT, as compared to the underlying stellar population (dots). The PNe colors are all extremely blue in F475W-F814W.

\section{Color magnitude diagrams}

Fig. 4 shows the location of PNe in some example CMDs. The PNe are very blue in the optical. Within a box with limits F474W-F814W from -2 to 0 , and F475W from 23.5 to 20.5 , roughly $70 \%$ of the objects are PNe. The other $30 \%$ may be compact HII regions, symbiotic stars, AGN, or previously unidentfied PNe.

\section{Ongoing work and concluding remarks}

We have models for the evolution of the emission lines in $\mathrm{PNe}$, derived from rough approximations for their dynamical evolution (Marigo et al. 2001, 2004, plus Cloudy (Ferland et al. 1998) for deriving emission line fluxes). These models have been reprocessed using version 8.01 of Cloudy and then used for synthetic photometry in the PHAT filters.

Table 1 lists the dominant emission lines that enter the PHAT filters, and Fig. 5 shows the synthetic spectra derived from Cloudy, with a $10 \AA$ resolution, for a solar-metallicity PNe model close to the peak of its [OIII] $\lambda 5007$ luminosity. We are now using these (and other) models to make predictions about the numbers and photometry of M31 PNe.

Besides the emission lines in Table 1, there are significant contributions from stellar, nebular H, and nebular He continuum in F275W and F336W, and significant Paschen continuum in $\mathrm{F} 814 \mathrm{~W}$.

We are also comparing the radial surface density gradient of $\mathrm{PNe}$ with other sources in M31's bulge (Rosenfield et al., submitted).

Our long term goal is to improve our understanding of the PNe progenitors and evolution. A crucial role in this project will be played by the spatially-resolved star formation history derived over $17 \mathrm{kpc}$ of the M31 disk (Williams et al. in prep.), which is expected 
Table 1. Partial list of relevant emission lines in PHAT filters

\begin{tabular}{lrrrl}
\hline ID & $\lambda(\AA)$ & $\log$ Flux $(\mathbf{c g s})$ & Flux $/$ Flux $(\mathbf{H} \beta)$ & Filter \\
\hline H I & 4861 & 34.568 & 1.0000 & F $475 \mathrm{~W}$ \\
H I & 4340 & 34.240 & 0.4698 & $\mathrm{~F} 475 \mathrm{~W}$ \\
H I & 4102 & 33.982 & 0.2597 & $\mathrm{~F} 475 \mathrm{~W}$ \\
H I & 3970 & 33.769 & 0.1592 & $\mathrm{~F} 475 \mathrm{~W}$ \\
H I & 12820 & 33.768 & 0.1585 & $\mathrm{~F} 110 \mathrm{~W}$ \\
He I & 10830 & 33.835 & 0.1853 & $\mathrm{~F} 110 \mathrm{~W}$ \\
He I & 10830 & 33.580 & 0.1029 & $\mathrm{~F} 110 \mathrm{~W}$ \\
He II & 4686 & 34.658 & 1.2305 & $\mathrm{~F} 475 \mathrm{~W}$ \\
He II & 3203 & 34.242 & 0.4725 & $\mathrm{~F} 336 \mathrm{~W}$ \\
He II & 2733 & 33.955 & 0.2442 & $\mathrm{~F} 275 \mathrm{~W}$ \\
He II & 2511 & 33.730 & 0.1453 & $\mathrm{~F} 275 \mathrm{~W}$ \\
He II & 10120 & 34.109 & 0.3481 & $\mathrm{~F} 110 \mathrm{~W}$ \\
O III & 5007 & 35.644 & 11.9229 & $\mathrm{~F} 475 \mathrm{~W}$ \\
O III & 4959 & 35.165 & 3.9611 & $\mathrm{~F} 475 \mathrm{~W}$ \\
S III & 9532 & 34.261 & 0.4941 & $\mathrm{~F} 110 \mathrm{~W}$ \\
\hline
\end{tabular}

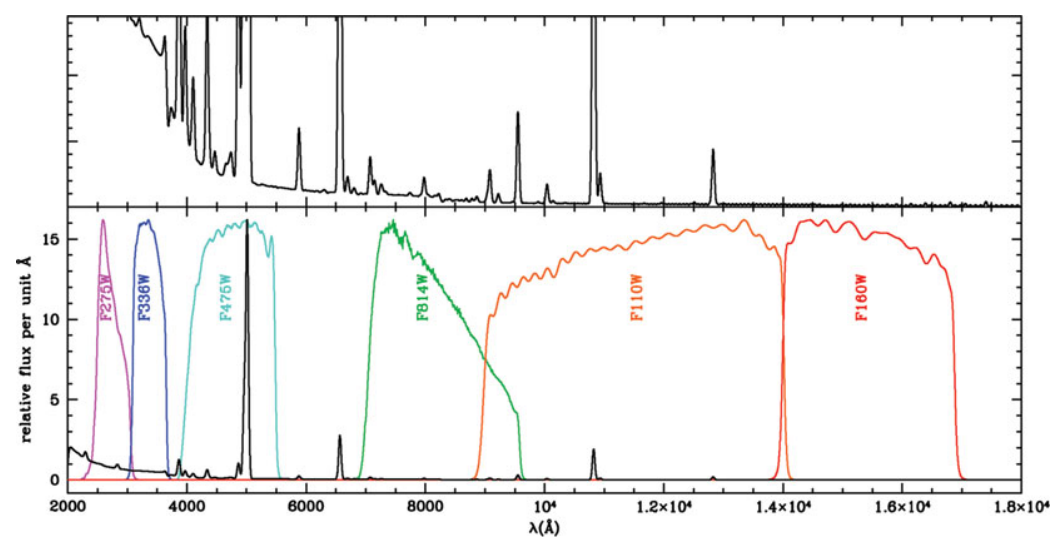

Figure 5. Model spectra of a PNe resulting from one of the Marigo et al. (2001) simple configurations combined with the photoionization code Cloudy, and compared to the transmission curves of PHAT filters. The top panel shows the same spectrum expanding the y-axis. The dominant feature in the spectrum is the [OIII] $\lambda 5007$ line falling in the F475W filter, but continuum emission does contribute at other wavelengths.

to correlate tightly with the specific numbers and observed properties of PNe. M31 represents a unique opportunity to study these correlations in a spiral galaxy similar to the MW.

To conclude: Although PHAT was not designed as a PNe survey, it provides (1) accurate astrometry and 6-filter photometry of known $\mathrm{PNe},(2)$ a good number of (possible) new detections in the F475W filter, (3) new insights between the relation between PNe and UV-bright stars in M31 Bulge (Rosenfield et al. submitted, and in prep.), (4) excellent prospects for studying the $\mathrm{PNe}$ in relation with their progenitors across the whole disk.

\section{References}

Ciardullo, R., Jacoby, G. H., Ford, H. C., \& Neill, J. D. 1989, ApJ, 339, 53

Ferland, G. J., Korista, K. T., Verner, D. A., et al. 1998, PASP, 110, 761

Marigo, P., Girardi, L., Groenewegen, M. A. T., \& Weiss, A. 2001, A\& A, 378, 958

Marigo, P., Girardi, L., Weiss, A., Groenewegen, M. A. T., \& Chiosi, C. 2004, A\&A, 423, 995

McConnachie, A. W., Irwin, M. J., Ferguson, A. M. N., et al. 2005, MNRAS, 356, 979

Merrett, H. R., Merrifield, M. R., Douglas, N. G., et al. 2006, MNRAS, 369, 120 